Reprod. Nutr. Dévelop., 1980, 20 (3 B), 827-841.

\title{
The social milieu of the developing monkey : Studies of the development of social perception
}

par L. A. ROSENBLUM, P. W. PAULLY Jr.

SUNY, Downstate Medical Center, 450 Clarkson avenue Brooklyn, New York 11203, USA

Summary. The study of the development of social behavior in two species of macaques, the pigtail (Macaca nemestrina) and bonnet (Macaca radiata), has revealed that social groups of these species present quite contrasting social milieus within which infants develop. Although infants of these species are confronted with social structures strikingly different in their interactive characteristics, it is of considerable adaptive significance for the infant, once moving freely in the environment to recognize, maintain orientation, and be able to move quickly towards the individual that consistently and effectively provides protection and sustenance. The research reported here concerns itself with the infants' emerging selectivity and differentiation in terms of social recognition and preference within their social structure.

The first study discussed, consists of the parallel assessment of infants' differential responses to their mothers when compared to familiar females and responses to their mothers when compared to strange females. This was accomplished by the utilization of a rectangular chamber with one-way glass placed at each end (to limit any subject-stimulus animal interaction). Infants in the mother-familiar female comparisons showed a significant overall preference for mother across the eight months of testing. However, in the mother-strange female comparisons, only eight of the twelve subjects met our minimum criterion of consistent differentiation of mother from stranger across the testing period. The nature of this differentiation was revealed in the highly significant sex by stimulus by replications effect in the analysis of variance. Male infants showed appreciably higher response to mother compared to stranger in the first replication compared to the second ; females on the other hand showed exactly the opposite pattern, significantly preferring mother on the second replication.

In order to proceed with an analysis of the stimulus basis of the infant visual recognition of mother, several studies were performed through the use of a medium within which manipulation of stimulus components was possible, i. e. color-videotape presentations. With this technique, images of various social stimuli could be controlled, reproduced across time and subjects, and handling of the subjects by the experimenters could be effectively limited. Three studies using video techniques are described : the first was a passive presentation to infant pigtails of videotapes consisting of their mothers, a familiar female and a strange female. Infants, placed in a rectangular chamber consisting of a clear plexiglass wall on one end (the rest being opaque) were shown these stimuli on an adjacent television monitor. Behavioral observations and vocalization analysis revealed a preference for mother over the other stimuli in regard to the distance at which they viewed her image and the type of vocalizations elicited by her image, with stranger being least preferred and familiar female falling between them.

The second video study provided the monkeys with operant control over the viewing of one or another of two color-videotaped images. The stimulus pairings of taped images 
involved comparisons of response to mother, familiar-female, stranger-female, familiar peer, stranger-peer, and stranger-male. In this setting, high viewing durations of mother occurred nearly twice as often, and similar viewing of familiar peer approximately 50 p. 100 more often than would be expected by chance; high duration scores to non-familiar peer appeared 33 p. 100 more often than chance expectancy with high viewing scores for the remaining stimuli at chance levels.

The final study described the responsiveness of group living bonnet macaques juveniles to an additional range of social stimuli presented via color-videotaped images. This experiment, tested the subjects in their home pens with their mother and another familiar mother-infant dyad. Three types of social stimuli were presented. They consisted of an unfamiliar adult female sitting passively, an unfamiliar adult male sitting passively and an unfamiliar adult male performing a series of open-mouth threat displays towards the subjects. The videotaped images of these stimuli repeatedly elicited meaningful picture and partner directed responses. Juveniles behaved submissively toward the threatening male stimulus and spent more time in contact with mother. On the other hand, the subject behaved more « assertively » to the female stimulus by approaching it and occasionally the male juveniles bounce-displayed during its presentations.

All studies reported illustrate the range of response of developing monkeys to the diverse network of social stimuli in their environment and speak towards the development of precise differentiation of mother from similar conspecifics and parallel discriminations among other members of their own natal group and others they may encounter.

Like the human child, the monkey enters into a distinct social relationship with its caregiver at birth ; the infant's limited functioning and consequent dependency makes such a relationship necessary for survival. However, it is not merely physical survival that the infant derives from this initial relationship with the primary caregiver. The entire course of the infant's subsequent social development is enacted within the social arena in which the mother functions and which in large measure molds her own behavior as well as that of the infant.

The major focus of our research has been the development of social behavior in two species of macaques, the pigtail ( $M$. nemestrina) and the bonnet ( $M$. radiata). To place the progression of behaviors described below into a broad developmental context, several basic phylogenetic and ontogenetic factors should be brought to the reader's attention. The macaque genus includes at least a dozen species and many subspecies, and these range in habitat accross more than 50 degrees of latitude, from seashore, swamp, and cliff to city, farm, and mountain. Mature body weight in males and females ranges rather broadly across the genus. A relatively narrow range of gestational periods averaging approximately 165 to 170 days seems to be characteristic of all macaques. Similarly, sexual maturity is reached in most macaque males at about 4 years and in females about 1 year earlier. These general paramefers of development, however, are often influenced by the conditions of research, with maturation progressing somewhat more rapidly in the laboratory than in the field.

Despite the common characteristics of the genus, our studies have revealed however that social groups of pigtails and bonnets present quite contrasting social milieus within which infants develop. When a group of wild-born pigtail macaques are placed in a relatively large laboratory pen, the adults disperse themselves throughout the available space, coming into close physical contact with one another only intermit- 
tently. On the other hand, when a similarly constituted group of bonnet macaques is placed in the same physical area, long periods of the day and evening are spent with numbers of individuals, often the whole group, in close physical contact with one another (Rosenblum, Kaufman and Stynes, 1964). This species difference in individual distance patterning is quite stable over a variety of changes in environmental conditions (Rosenblum, Kaufman and Stynes, 1969). Inasmuch as these patterns are also maintained following the birth of offspring, during development, infants of the two species are confronted with social structures strikingly different in their interactive characteristics.

Bonnet mothers, within moments of delivering their young, generally reestablish close contact with their associates and allow other members of the group to touch, explore, and even handle, the newborn. The pigtail mother on the other hand continues to remain relatively aloof and separated from other members of the group who attempt to establish some form of exploratory contact with her newborn. As a consequence of the difference in spacing patterns, and the less frequent restrictive and protective behavior of bonnet mothers, bonnet infants from birth onward consistently engage in more positive interaction with other members of the social group than do equivalently aged pigtail infants. Given the continued presence of these complex social units it seems reasonable to assume that in a slowly developing, largely defensive organisms, such as the monkey, there is considerable adaptive significance for the infant, once moving freely in the environment, to recognize, maintain orientation, and be able to move quickly towards, the unique caregiver in the environment that will consistently and effectively provide protection and sustenance. Our research of the last 5 years has convinced us that such recognition and preferential response, does not involve a rapid or immediate «imprinting 》 to the mother in the classical Lorenzian sense, but requires some form of gradual learning in keeping with more recent formulations of the development of filial responses in precocial birds (Hoffman, 1974).

Bearing in mind that infant rhesus are capable of simple discrimination learning by three weeks of life (Zimmerman and Torrey, 1965), Harlow and Harlow (1965) have suggested that in rhesus «there is every reason to believe that the infant learns attachments to a specific mother (the mother) long before our postulated reflex stage has past (n. b. 15-20 days)... » (p. 291). Although other informal observations (e. g., Bowlby, 1969) have also suggested very early discrimination and preferential response of macaques towards their mothers, these observations failed to distinguish between an infant's responsiveness to a specific individual and responses which are elicited by a specific class of behaviors, exhibited by an appropriate member of his species. A human infant, for example, may respond with visual orientation or a smile to a soft voice or to a smiling face, but such responses may not be specific to the mother, and do not meet the criteria of genuine attachment. Mothers become adept at providing their infants with such cures regularly, and thus the infant may appear to show genuine attachment. However, if it is only the mother who provides such cues, it would be a mistake to interpret the young infant's responses to her as a preferential response to her as a specific individual. In fact, there are indications in the nonhuman primate literature that under controlled conditions, monkey infants may neither discriminate the mother nor respond preferentially to her until after the first month of life. Jensen and Tolman (1965) studying pigtails, demonstrated that the macaque mother does 
not reliably discriminate her own infant from an infant of the same age until after the infant is two and half weeks of age. Furthermore, in the same study, even at thirty days of age, infants did not respond differentially to their own mothers. Takeda (1967) noted that in an hand-reared Japanese macaque « attaching behaviors » were directed to humans other than the caretaker until sometime after 80 days of the infant's life, and that the infant could readily be left with human strangers until that time. Of particular significance is an early study by Jensen and Tolman (1962) on two pigtail infants, each raised in isolation with its mother. These infants attempted «... to achieve the closeness customarilly experienced with their own mothers » (p. 134) when placed with a stranger at five and seven months of age respectively. It was not until several opportunities to interact with the stranger had elapsed before the infants « learned » to avoid the stranger. There is some reason to believe, therefore, that it may take as long as several months for macaque infants to learn to discriminate their mothers from other conspecifics and that rearing with her in isolation may retard such development, contrary to the speculation of some attachment theorists (Cairns, 1972 ; Hoffman, 1974). The process through which the infant comes to discriminate mother from nonmother is, of course, closely linked to the somewhat pocrly circumscribed concept of infant attachment. Although different elements of behavior may be part of the attachment pattern at varicus times of life, it is generally accepted that early in life at least, the attachment concept involves the tendency of the young to seek the proximity of certain other members of their species (Ainsworth, 1969; Schaffer and Emerson, 1964). Furthermore, there has been increasing agreement that the behaviors subsumed under the attachment concept, a) are limited to those which are directed towards parficular individuals rather than towards a class of conspecifics ; b) are directed towards those selected individuals during a protractive segment of the infant's life ; and, c) must emerge in a variety of situations and not be specific to any particular setting (Ainsworth, 1969 ; Rosenblum and Alpert, 1974).

Previous research regarding the development of attachment in nonhuman primates, has provided a wealth of comparative data in recent years, but has also left several important dimensions of differentiation and selectivity unexplored. Thus, Sackett in a series of early studies, using a « selection circus », presented considerable evidence regarding the emergence of social preference in rhesus monkey infants on the basis of species, age, gender, and familiarity factors (Sackett, 1970, 1972). However, the opportunity for subjects and stimulus animals to interact during tests in this apparatus provides some ambiguity regarding the basis for differential responses observed in these studies. Similarly, studies of infants' response to mothers and others in squirrel monkeys, while indicating preferential response of older infants to mothers under some conditions, presented a similar problem (Kaplan, 1972).

In prior studies carried out in our own laboratory, the problems of subjectstimulus animal interaction during preference tests were overcome by utilizing a one-way glass partition which allowed subjects to see the social stimuli but not viceversa (Alpert and Rosenblum, 1974 ; Rosenblum and Alpert, 1974, 1977). These studies involved group-reared bonnet and pigtail macaques as well as «single-dyad» reared bonnet macaque infants. «Single-dyad » rearing involves removal of a mother and her infant, within hours of birth, to a separate pen where the dyad lives alone thenceforth. 
Preferential response towards mother was found to develop by approximately three months of age in the group-reared macaque. In addition, sex and species differences in the patterns of preference behavior were observed. The behavior of female bonnets suggested that they formed specific attachment to the mother earlier and more intensely than did male bonnets. Furthermore, group-reared bonnet macaques of both sexes showed stronger attachment to mother than group-reared pigtails of either sex. It is particularly striking that, in keeping with Jensen and Tolman (1962), the single-dyad infants failed to show any consistent preference for mother compared to a stranger until after the infants reached 8 months of age, i. e. about 5 months later than the group-reared infants.

However, the initial studies, particularly of group-reared infants, still failed to come to grips with two important issues. First, it is possible that some aspects of the patterns of attachment behavior shown in those studies were not a reflection of recognition of mother as a unique object ; rather, responsivity may have been based on the fact that mother was simply more familiar than stranger. A second concern derives from the fact that under normal circumstances, the infant's initial task is to differentiate mother from other familiar females. Certainly the frequency of inter-group encounters suggests that differentiation of familiar (own-group) from stranger (othergroup) may well be important for the developing macaque; similarly, recent theory and evidence suggests the importance of kin-nonkin differentiation (e. g. Kurland, 1977 ; Wu and Holmes, in press) ; nonetheless, the differentiation of response to familiars and unfamiliars must at least be accompanied by the emergence of selectivity of infant response to mother as opposed to other quite familiar adults. In an attempt to clarify some of these ambiguities, a study was devised which allowed parallel assessment of infants' responses to their mothers when compared to familiar females and responses to their mothers when compared to strange females (Alpert, 1978).

Subjects.

Subjects were 12 bonnet macaque infants (Macaca radiata), 6 males and 6 females, conceived and born in the Primate Behavior Laboratory of the Downstate Medical Center. Mothers were socially reared, multiparous females. Within 24 hours after the birth of the infant, each mother-infant dyad was removed from the breeding group and placed in an individual pen. When four dyads, including two male and two female infants born within three weeks of one another were obtained, the special rearing conditions of this study were implemented.

\section{Rearing Conditions.}

The infants were reared in an «alternating double-dyad situation » such that each infant lived alternately with its mother and, 1) a same-sex infant-mother dyad for two weeks, and, 2) an opposite-sex infant-mother dyad for two weeks. In the third two-week period, the original same-sex dyad was returned and these alternations were repeated throughout the study.

Testing.

All testing was carried out in a chamber $2.5 \mathrm{~m}$ long, $0.5 \mathrm{~m}$ wide and $0.6 \mathrm{~m}$ high. The interior of the chamber was painted flat black and the floor covered with shaggy 
carpeting to facilitate locomotion in young infants. The top of the chamber was covered with hinged Plexiglas lids covered with fine mesh screening to prevent the infants from seeing out of the test chamber. The center $0.6 \mathrm{~m}$ area of the chamber was enclosed by two guillotine Plexiglas doors which could be raised and lowered remotely by the observer. The infant was placed into this start area through a small door on the side wall of the chamber. Actual observation of the interior of the chamber, with minimal distraction for the infant, was made possible by a large mirror hung at a 45 degree angle $1.0 \mathrm{~m}$ above the chamber itself. Of critical significance to the operation of the test procedure were the two end walls of the chamber, each of which contained a large one-way vision glass window. No illumination was provided within the chamber itself, whereas each stimulus chamber was brightly illuminated. Thus, because of the one-way glass and differential lighting, the infant was easily able to see both the mother and the stranger or the familiar, but stimulus animals could not see the infant within the test chamber. Thus differential response to the infant by the stimulus animals was effectively eliminated; this insured that the infant was responding primarily to the visual appearance of the social stimuli rather than to the specific behaviors directed toward it by either animal.

\section{Social Preference Testing.}

During one week each month, each infant received two days of preference testing in the apparatus, spaced one day apart. As part of a comprehensive program of testing the infants' response to various stimuli, several different types of trials were presented each day. The comparison trials of mother versus familiar female and mother versus strange female were presented once on each of the two test days, with the order of trial presentation randomized and the location of stimuli balanced across replications. Each trial lasted $150 \mathrm{sec}$ with a two-minute inter-trial interval. At the beginning of each trial, the infant was placed in the start chamber for $30 \mathrm{sec}$ before the Plexiglas doors were raised. When the doors were raised, the trial began. The total amount of time an infant spent in the $30-\mathrm{cm}$ segment next to the stimulus chamber at each end of the apparatus was recorded by means of a push-button clock device ; this recorded duration was designated as «choice-time».

The two days of apparatus testing occurred during the second week that the infant was living with the same sex peer and its mother. This female served as the "familiar-female » for that infant throughout the eight months of testing. For females and males the average age at which testing began was 3.8 weeks of life. Each subsequent test was exactly four weeks after the previous one and continued until the infants reached 8 months of age.

Two additional mother-infant pairs were reared similarly to the 12 subjects and their mothers. One infant was a male and one was a female. They were approximately the same age as the test subjects and were also reared in a double-dyad situation. These two mothers served as the strange females for all tests.

Based on previous work, as an additional means of assessing the development of preferences, a minimum preference criterion was established as follows : if an infant spent at least $10 \mathrm{sec}$ more in proximity to mother compared, to the stimulus to which she was compared for two consecutive months, the first of these two months was judged to be the age at which that particular preference was established for that subject. 


\section{Results.}

Across the eight months of testing, infants spent an average of $82.5 \mathrm{sec}$ i. e. $55 \mathrm{p}$. 100 (sd $=0.05$ p. 100) of both mother-stranger and mother-familiar female comparison trials, in the two choice areas at the stimulus ends of the apparatus. However, the patfern of preferential response in the two comparison conditions differed rather markedly, particularly after the second month of life.

Response to mother, in the mother-familiar female comparison trials, was not significantly greater in any particular month. Nonetheless, subjects showed a significant overall preference for mother $(F=29.0 ; d f 1 / 10 ; p<0.01)$ cicross the eight months of testing, spending an average of $55 \mathrm{sec}$. next to mother and only $27 \mathrm{sec}$ next to the familiar female. Application of the minimum preference criterion revealed that several subjects began to shown preference for mother as early as the first month and a majority of subjects met the criterion by the second month of life when an average of 63.2 p. 100 of total response time was spent next to the mother. No sex differences emerged in these trials, but a significant replications effect in the analysis $(p<0.05)$ reflected the tendency of subjects to respond more strongly to mother on the first, as compared to the second, replication trials.

Infants' response in the mother-strange female comparison trials was appreciably more complex than in the mother-familiar female comparisons. Consistent differentiation of mother and stranger did not emerge in many subjects! Unlike the response to mother, when she was compared to the familiar female, analyses revealed that subjects showed no significant preference for mother compared to stranger across the first eight months of life $(F=4.0 ; d f=1 / 10 ; p<0.05)$. Indeed, only eight of the 12 subjects met our minimum criterion of consistent differentiation of mother from stranger across the testing period. Infants spent only 52.9 p. 100 of their total choice time with mother in months- 14 in this comparison. Although subjects average $48.4 \mathrm{sec}$ per trial (59.5 p. 100 of choice time) to mother and $33.0 \mathrm{sec}$ to stranger in months 5-8, even these levels of response were not statistically reliable, and the somewhat ambiguous nature of this pattern was revealed in the highly significant sex by stimulus by replications effect in the analysis of variance $(F=26.7 ; d f=1 / 10 ; p .<0.01)$. As in the mother-familiar comparisons, males showed appreciably higher response to mother compared to stranger in the first replication compared to the second ; females on the other hand, showed exactly the opposite pattern, failing to differentiate mother from stranger on the first replication but significantly preferring her on the second.

The differences in response to mother in the two types of trials were borne out in comparisons of scores to mother under the two conditions and comparisons of response to stranger and familiar female when each was paired with mother. Mean levels of response to mother when paired with familiar female was $55 \mathrm{sec}$ per trial while response to her when compared to stranger averaged only $46 \mathrm{sec}$ per trial $(F=10.5$; $d f=1 / 10 ; p<0.01)$. A significant stimulus by replication effect $(p<0.01)$, revealed that whereas response to mother in the mother-familiar trials was generally greater than in the mother-stranger trials, this difference was significantly larger in the first of the two replications. No significant sex or age effects emerged in these comparison analyses. Further analyses indicated a significantly greater response to stranger 
(Mean $=36.0 \mathrm{sec})$ as compared to familiar female (Mean $=27.0 \mathrm{sec} ; F=2.2$; $d f=1 / 10 ; p<0.01)$ with much of this difference reflected in the first replication scores $(p<0.05)$. Perhaps most significant of all was the fact that whereas 11 of the twelve subjects met criterion levels of differentiation of mother and familiar female by month six, only eight subjects met this criterion in the mother-stranger trial throughout testing. Indeed the only subject failing to meet the criterion in mother-familiar female trials also failed to do so in the mother-stranger condition.

This study, taken in conjunction with the results of our prior work, strongly suggests that $: a$ ) the infant monkey slowly develops the capacity to differentiate mother from nonmother (most subjects require 2-3 months for visual differentiation) ; $b$ ) the differentiation process, although perhaps genetically constrained, depends upon the opportunities the infant has to make direct comparisons between the appearance of mother and others in its rearing environment (the infants of the more gregarious, permissive bonnets become selective earlier and more completely than the more dispersed, restrained pigtails ; single-dyad infants are extremely delayed in developing differentiation) ; and, $c$ ) differentiation proceeds from the « particular » to the " general », i. e. the infant, in a sense, first learns « who the mother is not», and only later acquires, perhaps in a learning-set fashion, the class distinction, «nonmother » (mother is relatively rapidly distinguished from a familiar, while differentiation of mother from stranger takes considerably longer in double-dyads, but is acquired more rapidly when infants are bonnet-group reared).

This work has all focused on the use of vision as the basis for differentiation. Of course, in the neonate other sensory systems may well be the primary means of identification (Kaplan, 1977).

Nonetheless, as the infant moves from the mother with increasing independence, entering more autonomously the physical and social world that surrounds it, it is through vision that the infant maintains its «life-line » with the mother; whether in play or while foraging, the semi-independent infant engages in frequent visual checks on its mother's location ; when in danger the infant's flight to the mother must be direct and guided by distal location cues, which can involve audition, but depend most heavily on vision.

What is the nafure of those cues which allow visual differentiation of mother from others? One cannot readily alter the appearance of the living mother in meaningful ways, nor induce her to behave or contort her body in an unusual manner. Since we are interested in the infant's response to particular individuals, we certainly cannot use stuffed or dummy versions of a " generalized 》 mother-monkey, in the manner of classical ethology. However, if one could establish selective responsivity of infants to the visual cues of mother in a medium within which manipulation was possible, the analysis of the basis of infant recognition could proceed.

With this objective in mind, we have recently begun a program involving the use of color-videotaped images of various social stimuli to which infant responses may be reliably assessed. These video-stimulus techniques allow controlled regulation of stimuli, reproducibility across time and subjects, and minimal handling and use of animals that are also subjects of the experiment as stimulus animals for others.

In the future, as the effectiveness of these video methods is explored further, through the use of special-effects generators and tape-blending techniques, we should be able to alter stimulus images in ways that will allow determination of the spe- 
cific cues involved in recognition of mother, familiars, peers, conspecific and other salient dimensions of the social nexus. In the first study of infant response to video stimuli, five pigtail infants, approximately 7-12 months of age, served as subjects. These infants were raised in a double-dyad situation described above. Each infant had undergone a six week separation from mother and the familiar female, which concluded 2-3 months prior to these tests. One infant of that study died during separation. Color video recordings were made of each mother and familiar female in the home pen and five-minute edited tapes, meeting visibility and stable, nonthreatening behavioral criteria, were created. Because of the living arrangements, the same tape of a given female served as the mother stimulus for one infant, the familiar stimulus for another and as the stranger stimulus for a third infant.

For testing with the color videotaped images, the infants were placed in a steel chamber $(1.2 \mathrm{~m} \times 0.54 \mathrm{~m} \times 0.67 \mathrm{~m})$ in which both sides and the back were opaque and the front wall was constructed of clear Plexiglas. The floor was covered with $0.6 \mathrm{sq} \mathrm{cm}$ wire mesh. Four 40 watt fluorescent bulbs illuminated the interior of the chamber through the mesh top. The interior of the chamber was demarcated into three distance zones, each $0.4 \mathrm{~m}$ in length running from the front to the back of the chamber. A $58 \mathrm{~cm}$ (diagonal) color-video monitor (Zenith H2310W) was placed $61 \mathrm{~cm}$ directly in front of the chamber. The $41-\mathrm{cm}$ high picture tube allowed presentation of the images of the stimulus animals in their actual dimensions. Above the color-video monitor was placed a video camera (Fairchild TC-175) through which both monitoring and recording of the subject's behavior and movements was done. Two black and white video monitors in an adjacent room were used to monitor the recording of the subject's trial, and to monitor the videotaped stimulus being presented. Sound recordings of the subjects were made using the audio tracks of the videocassette recorder (JVC-6060 U-3/4") through a microphone (Claricon 38504) centrally situated beneath the apparatus. Presentation of the color videotaped stimuli was accomplished by means of a $3 / 4^{\prime \prime}$ videocassette player (JVC-5200 U). A series of behaviors relating to infant effect (e. g. toe curling, grimace, crouch ; Rosenblum and Plimpton, 1979) were scored as well as infant orientation and location in the chamber throughout the trial. Each infant received three trials per day for three test days in counterbalanced order. Trials were separated by $5 \mathrm{~min} ; 5 \mathrm{~min}$ of adaptation began each day's test.

Preliminary analyses of the data from this study indicate that four broad types of vocalization were emitted by the infants in response to the video stimuli : a) Coo; a moderately high amplitude call, in most cases involving a single uniform frequency in the $1000 \mathrm{~Hz}$ range ; b) Rasp-Coo ; a biamplitude call, involving a fairly uniform single frequency in the $1750 \mathrm{~Hz}$ range, in which high and low amplitude elements of the basic frequency are rapidly alternated ; c) Screech ; these high amplitude calls are noisey in character and fall into two primary frequency ranges of either 3000 or $4750 \mathrm{~Hz}$; and, d) other calls ; these varied call types, which appeared least often, involved several recognizable types and complex mixtures; the more discrete types included : chirp (low amplitude, $4000-6000 \mathrm{~Hz}$ ), Kwah (low amplitude, short bursts in the $1750 \mathrm{~Hz}$ range), Raah (moderate amplitude, short bursts at about $250 \mathrm{~Hz}$ ), and Whoa (low amplitude, $750-850 \mathrm{~Hz}$ range). Figure 1 shows the total percent of each trial that subjects spent vocalizing during each type of presentation : most vocalization time occurred in the presence of mother, least in the presence of stranger; in 14 of the 
15 within trial comparisons across the 5 subjects, mother scores were greater than those to strangers; similarly, for four subjects, in all 12 trials, vocalization time to mother was greater than that to familiar. It is noteworthy that one infant, that had been severely mauled by its familiar female several months earlier, vocalized almost incessantly (primarily with " other » calls) in her " presence » and its total vocal time (74 p. 100) to familiar was the highest single score observed. The breakdown of vocalization types revealed the most consistent differentiation in terms of the high intensity « rasp-coo» and «screech» (fig. 1) Four of the subjects showed more frequent vocalizations of these types to mother than to familiar or stranger on every trial. "Cooing » on the other hand was more variable; the one subject that showed fewer high intensity calls to mother consistently « cooed » more to her than either stranger or familiar on each trial.

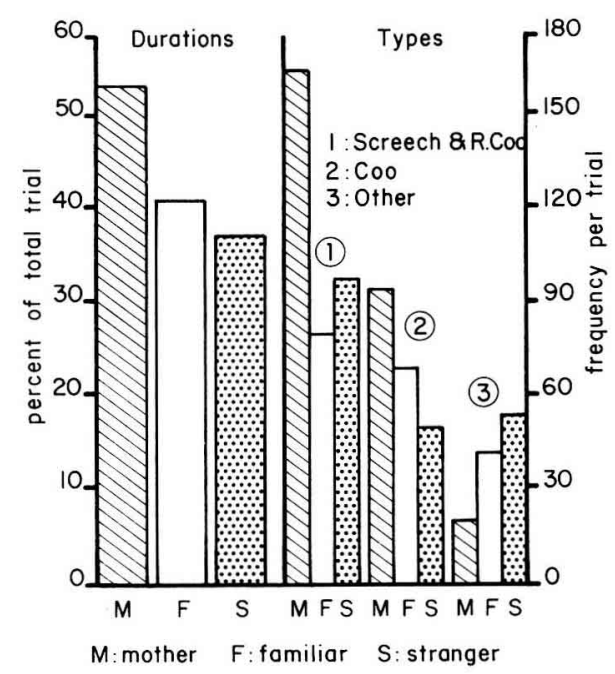

FIG. 1. - The total duration of vocalization to video images of mother $(M)$, familiar female (F) and stranger female (S) ; Right side : the frequency of each major type of vocalization to each of the three video stimuli.

The remaining subjects, although cooing somewhat more to mother and least to stranger, were not consistent in cooing from trial to trial, particularly with regard to the comparison of scores to mother and familiar female. The more variable element, " other » calls, although generally less frequent, was quite consistently emitted least often in response to the mother stimulus and most often to the stranger. (It should be noted that future work, involving computer-based zero-crossing discriminant analyses, may well allow identification of more specific call-stimulus relationships.)

Analysis of the subject's location in the chamber during test trials revealed consistent differences in response to mother, familiar and stranger as the trial progressed. As reflected in figure 2, although the infants began each trial at approximately the same distance from the video image, by the end of the trial, they moved nearly half as far from the mother and twice as far from the image of the stranger. Average positions with regard to the familiar female stimulus fell ambivalently between those recorded for mother and stranger stimuli.

Although the data analyses of this study are not yet complete, it is evident from the findings described above that infant monkeys can indeed make meaningful differenta- 
tions of their mother, familiar females and strangers on the basis of visual cues contained in color-video stimuli.

FIG. 2. - The average distance from each of the video stimuli during successive minutes of the first replication trials.

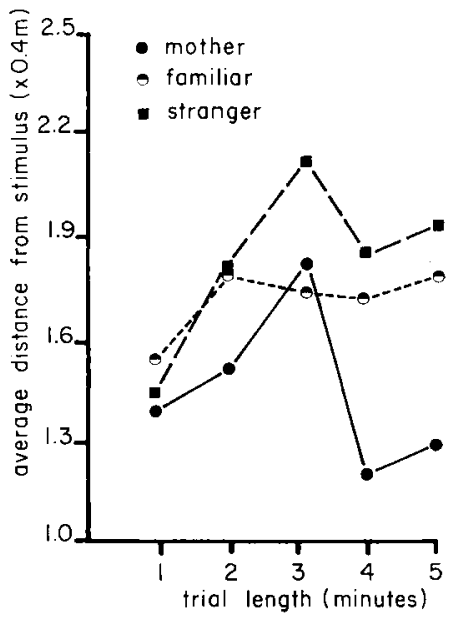

This fact has also been demonstrated in a related study involving a group of five pigtail juveniles, each approximately 2.5 years of age. This latter study involved the use of color-video stimuli in an operant situation, similar to that employed by Humphrey (1972). Given the opportunity to view one or another of two taped images each time a lever was depressed, several consistent viewing preferences between particular pairings were recorded for most of these subjects (See fig. 3). The stimulus pairings, replicated 8 times each, involved comparisons of response to mother, familiarfemale, stranger-female, familiar-peer, stranger-peer, and stranger-male. Although response durations were variable across subjects and trials, considering the range of operant response within each subject, high viewing durations of mother occurred nearly twice as often, and similar viewing of familiar peer about 50 p. 100 more often,

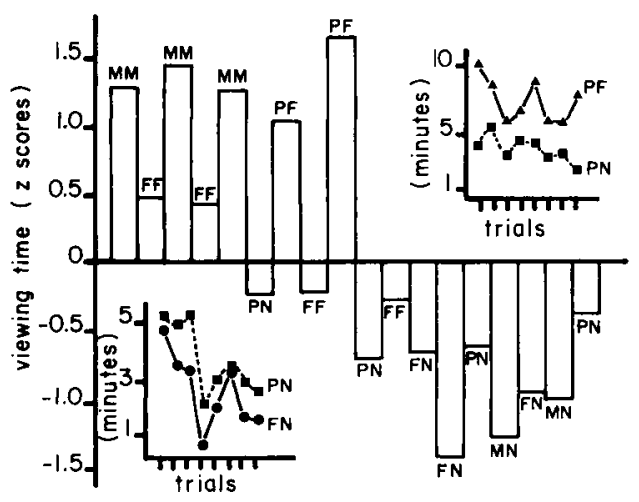

FIG. 3. - The average duration of viewing time in subject \# 5 to various videotaped social stimuli expressed as z scores; actual pairings may be read from left to right : Code $M M=$ mother ; $F F=$ familiar female ; $P F=$ familiar peer $; P N=$ non familiar peer ; $F N=$ nonfamiliar female $; M N=$ nonfamiliar male. Inserts : Duration of response to familiar and nonfamiliar peer and to non familiar peer and nonfamiliar female on successive replication trials. 
than would be expected by chance; on the other hand, high duration scores to non familiar-peer appeared only $1 / 3$ as often as chance expectancy. High operant viewing scores for the remaining stimuli were at chance frequencies. Thus, although the situational and motivational factors influencing operant performance for social stimuli are poorly understood at present, young monkeys can distinguish between familiar and unfamiliar social partners of various classes via video images, and will seek or maintain those stimuli at different levels.

In an effort to explore the efficacy of the video techniques even further, in the last of the current series of studies, conducted by Plimpton, Rosenblum and Swartz in our laboratory, we investigated responsiveness of group living bonnet macaques juveniles to an additional range of social stimuli presented through color videotaped images. Unlike the previous studies involving apparatus, in this experiment, group reared juvenile bonnets were tested in their home pens with their mother and another familiar mother-infant dyad.

The subjects of this study were twelve 2.5 year old juvenile bonnet macaques (M. radiata), six males and six females, that had been born in the laboratory and were living in three different groups with four mother-infant dyads in each. These subjects had never lived with an adult male. They were housed and tested in large pens, $2.1 \times 1.8 \times 2.6 \mathrm{~m}$, which contained two shelves along the rear wall. The front of each pen contained two windows ; one, made of one-way vision glass was used for behavioral observations; the other, composed of clear Plexiglas, was used for the presentation of color videotape recordings appearing on a $63.5 \mathrm{~cm}$ (diagonal) color television set placed against the window. In addition, on the floor of each pen, a connected series of $11 \mathrm{~cm}$ high ceramic bricks demarcated a $1.2 \times 1.5 \mathrm{~m}$ " approach zone » surrounding the video presentation window.

Each test trial included the color videotape representations of three types of social stimuli : (1) an unfamiliar adult bonnet female stitting quietly, designated the " passive female »; (2) an unfamiliar adult bonnet male sitting quietly, designated the " passive male »; and (3) an unfamiliar adult bonnet male directing a series of 13 openmouth threat displays toward the subjects, designated the "threatening male » (Kaufman and Rosenblum, 1966). Each social stimulus sequence was $150 \mathrm{sec}$ in duration, edited from original tapes of longer duration. These sequences were preceded, separated and followed by a 300-sec blank tape segment. The order of presentations was balanced across trials, and observers were blind regarding wihch social stimulus was being presented at any given time.

For testing, two juveniles and their mothers were removed from their group and the remaining two dyads, containing juveniles of the same sex, were then observed during a $27.5 \mathrm{~min}$ test trial preceded by a 5 - $\mathrm{min}$ habituation period. The second pair of same-sex juveniles and their mothers were then tested. Testing was done each week for 3 consecutive weeks.

Observations were made by two observers who recorded the frequency and duration of both partner- and picture-directed responses during each stimulus presentation. In addition, social interactions were also recorded in the $150 \mathrm{sec}$ prior to each stimulus presentation. Although a variety of behaviors were initially recorded, the following four patterns of response occurred most frequently : (1) Approach - the frequency of entering and duration of remaining in the " approach zone »; 
(2) Lipsmack - the frequency of discrete bouts of picture-directed lipsmacking ; (3) Bounce-display - vigorous shaking and/or bouncing on the inanimate environment; (4) Social contact - any type of physical contact between subjects lasting longer than $3 \mathrm{sec}$. For purposes of data analysis, the mean score per subject across the three trials was used in a two-way analysis of variance (Sex $\times$ Stimulus) with post hoc comparisons made using the Duncan New Multiple Range Test (Duncan, 1955.)

It is worth noting that the range of socially directed behavior which appeared during stimulus presentation was quite limited. Perhaps as a dual consequence of the partial disruption of the social group, which the experiment involved, and the focus ing of attention on the video stimuli, a number of behaviors such as grooming, aggression and play, which were scored originally did not occur with sufficient frequency to be analyzed.

Presentation of video stimuli produced immediate alerting and orientation of the juvenile subjects of this study and then varying response as a function of the type of stimulus. As reflected in figure 4 the video image of the passive female was approached most frequently while that of the threatening male was approached least $(F=14.06$; $d f=2 / 10 ; p<0.01)$. Although the passive female and threatening male stimuli differed dramatically in the frequency of approaches elicited $(p<0.005)$, the intermediate levels of response to the passive male stimulus were not significantly different from the other two. There were no sex differences in these frequency of approach scores. Analysis of the time spent in the approach zone while stimuli were presented followed an essentially similar pattern, except that male juveniles spent significantly less time in the zone across all conditions $(\bar{X}, 104 \mathrm{sec})(F=8.31 ; d f=1 / 10 ; \mathrm{P}<0.05)$. The mean duration of approach to the passive female, passive male and threatening male stimuli were 48, 16 and 11 seconds respectively $(F=24.26 ; d f=2 / 20 ; p<0.01)$. Again, there was no significant sex of subjects $x$ stimulus interaction effect. An interesting sex difference did emerge in the appearance of bounce-display. Four out of the six males performed at least one bounce-display during the presentation of the passive

FIG. 4. - The average duration of approach to each of the 3 video stimuli in the social test sifuation.

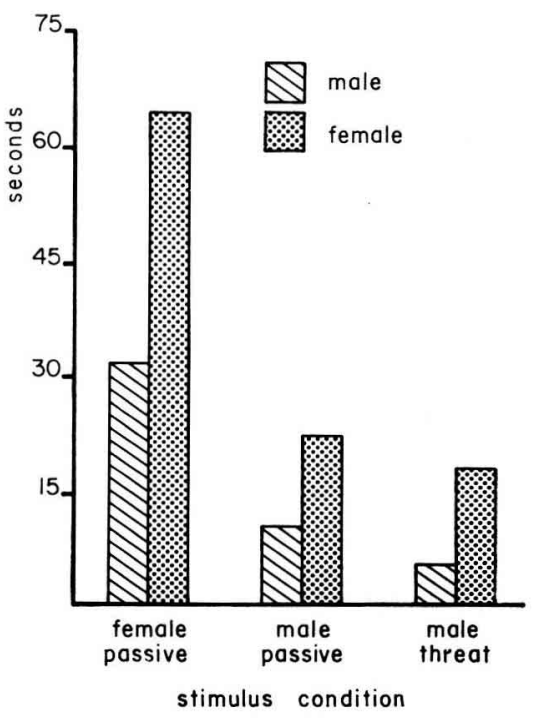


female stimulus, whereas none of the female subjects did. Indeed females failed to bounce display at any time.

Two forms of social behavior occurred with greatest frequency during the threatening male sequence. The frequency of lipsmacking, directed at the video stimuli, differed significantly across conditions $F=7.47 ; d f=2 / 20 ; p<0.01$ ). Lipsmacks, generally considered a submissive or appeasment gesture, were elicted by the threatening male more than twice as often as the passive male $(p<0.05)$ and more than four times as often as the passive female $(p<0.05)$. Once again, despite intermediate mean levels of response to the passive male, the latter condition did not differ significantly from the passive female condition. There were no sex differences in the rate of lipsmacking to the stimuli.

Finally, an examination of the infant-mother interaction revealed that the time that the subjects spent in contact with their mothers also varied significantly across the stimulus conditions $(F=3.48 ; d f=3 / 30 ; p<0.05)$ with scores on this measure at their highest level during the threatening male condition. In the $150 \mathrm{sec}$ prior to the onset of the taped social stimuli, the juveniles were in contact with their mother an average of $22.9 \mathrm{sec}$ and stayed at similar levels during the passive female $(\bar{X}=21.1)$, and passive male $(\bar{X}=25,4)$ presentations. However, the presentation of the threatening male stimulus resulted in significantly more contact with mother $(\bar{X}=45.7)$, than either the prestimulus period, the passive female or the passive male conditions $(p<0.05)$. The increase in contact scores was selectively directed towards mother. There were no significant differences in contact scores with either the familiar peer or the familiar nonmother adult female across the stimulus conditions. Thus, the videotaped images of the three social stimuli repeatedly elicited meaningful picture and partner-directed responses. When the threatening adult male stimulus was presented, despite their lack of prior experience with males, the juveniles behaved submissively toward it and spent more time in contact with mother. In contrast, they behaved more "assertively 》 to the female stimulus by approaching it and, in some cases, the male juvenile bounce-displayed during its presentations. These behaviors are consistent with patterns we observe in other contexts involving live animals.

These studies, taken together, illustrate the range of response of developing monkeys to the diverse network of social stimuli within which the infant-mother dyad functions. The selectivity of response demanded of infants as they achieve increasing autonomy, requires the development of precise differentiation of mother from essentially similar conspecifics and parallel discriminations amongst other members of their own natal group and other troops they encounter. Experience regarding the diversity and intimacy with which infants encounter other animals in various settings appears to influence the rate and clarity with which such differentiations are acquired. Although the processes involved in attaining these distinctions undoubtedly involve a complex mixture of phylogenetic and ontogenetic factors, our inability to achieve precise, manipulative stimulus control in the past has hampered efforts to disambiguate the diverse cues involved in mediating this crucial developmental phenomenon. It is in this regard that the use of a variely of color-video techniques, such as those described above, should help us to discern the stimulus factors which control the emergence of patterned social perception. 


\section{References}

AINSWORTH M. D. S., 1969. Object relations, dependency, and attachment : A theoretical review of the infant-mother relationship. Child Develop., 40, 969-1025.

ALPERT S., 1978. The response of infant monkeys to mothers, familiars, strangers, and peers : Development during the first eight months of life (Unpublished $\mathrm{Ph}$. D. Thes.).

ALPERT S., ROSENBLUM L. A., 1975. The influence of gender and rearing conditions on attachment and response to strangers. Symp. 5th Congr. int. Primat. Soc., 218-231.

BOWLBY J., 1969. Attachment and loss : Attachment. Basic Books, New York.

CAIRNS R. B., 1972. Attachment and dependency : a psychobiological and social learning synthesis, 29-80. In GERWIRTZ J. L., Attachment and dependency, W. H. Winston and Sons, Washington, D. C.

DUNCAN C. B., 1955. Multiple range and multiple F tests. Biometrics, 11, 1-42.

HARLOW H. F., HARLOW M. K., 1965. The affectional systems, 287-334. In SCHRIER A. M., HARLOW H. F., STOLLNITZ F., Behavior of nonhuman Primates, II, Acad. Press, New York.

HOFFMAN H. S., 1974. Fear mediated processes in the context of imprinting, 25-48. In LEWIS M., ROSENBLUM L. A., Origins of fear, John Wiley and Sons, New York.

HUMPHREY N. K., 1972. "Interset » and «Pleasure »: Two determinants of a monkey's visual preferences. Perception, 1, 395-416.

JENSEN G. D., TOLMAN C. W., 1962. Mother-infant relationship in the monkey, Macaca nemestrina : The effect of brief separation and mother-infant specificity. Journ. comp. Physio. Psych., 55, 131.

JENSEN G. D., TOLMAN C. W., 1965. Mother-infant relationship in the monkey, Macaca nemestrina : Development of specificity of maternal response to own infant. Journ. Comp. Physio. Psych., 59, 305-308.

KAPLAN J. N., 1972. Differences in the mother-infant relations of squirrel monkeys housed in social and restricted environments. Devel. Psychobiol., 5, 43-52.

KAPLAN J. N., CUBICIOTTI D. D., REDICAN W. K., 1977. Olfactory and visual differentiation of synthetically scented surrogates by infant squirrel monkeys. Devel. Psychobiol., 12, 1-10.

KURLAND J. A., 1977. Kin selection in the Japanese monkey, 1-143. In SZALAY F. S., Contributions to primatology, Karger, Basel.

PLIMPTON E. H., ROSENBLUM L. A., SWARTZ K. B., 1979. Response of juvenile bonnet macaques to social stimuli presented through color videotapes. Devel Psychobiol. (in press).

ROSENBLUM L. A., ALPERT S., 1974. Fear of strangers and specificity of attachment in monkeys, 165-193. In LEWIS M., ROSENBLUM L. A., Origins of fear, John Wiley and Sons, New York.

ROSENBLUM L. A., ALPERT S., 1977. Response to mother and stranger : A first step in socialization, 463-478. In CHEVALIER-SKOLNIKOFF S., POIRIER F. E., Socialization in primates, Aldine, New York.

ROSENBLUM L. A., KAUFMAN I. C., STYNES A. J., 1964. Individual distance in two species of macaque. Anim. Behav., 12, 338-342.

ROSENBLUM L. A., KAUFMAN I. C., STYNES A. J., 1969. Interspecific variations in the effecis of hunger of diurnally varying behavior elements in macaques. Brain, Behav. Evol. 2, 119-131.

SACKETT G. P., 1970. Unlearned responses, differential rearing experiences, and the development of social attachments by rhesus monkeys, 112-140. In ROSENBLUM L. A., Primate behavior : Developments in field and laboratory research, Acad. Press, New York.

SACKETT G. P., 1972. Exploration behavior of rhesus monkeys as a function of rearing experiences and sex. Develop. Psych., 6, 260-270.

SCHAFFER H. R., EMERSON P. E., 1964. The development of social attachments in infancy. Monogr. Soc. Res. Child Develop., 29, 3, (Serial \# 94, whole issue).

TAKEDA R., 1967. Development of vocal communication in man-raised Japanese monkey II. Primates, 7, 73-116.

WU H. M. H., HOLMES W. G. Kin preference in infant Macaca nemestrina. In BARLOW, G. W., SILVERBERG J., Sociobiology : Beyond nature-nurture. D. C. AAAS Publ., Washington, D. C. (in press).

ZIMMERMAN R. R., TORREY C. C., 1965. Ontogeny of learning, 405-477. In SCHRIER A. M., HARLOW H. F., STOLLNITZ F., Behavior of nonhuman primates, II, Acad. Press, New York. 